\title{
ChemComm
}

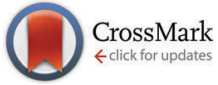

Cite this: Chem. Commun., 2016, 52, 9093

Received 5th January 2016 Accepted 25th February 2016

DOI: 10.1039/c6cc00095a

www.rsc.org/chemcomm

\section{A highly selective fluorogenic probe for the detection and in vivo imaging of $\mathrm{Cu} / \mathrm{Zn}$ superoxide dismutase $\uparrow$}

\author{
Liyun Zhang, $\ddagger^{*^{a}}$ Jun Cheng Er, tbc $^{\text {bc }}$ Ho Jiang, ${ }^{d}$ Xin Li, ${ }^{a}$ Zhaofeng Luo, \\ Thomas Ramezani, ${ }^{e}$ Yi Feng, ${ }^{e}$ Mui Kee Tang, ${ }^{b}$ Young-Tae Chang ${ }^{b}$ and \\ Marc Vendrell*e
}

\begin{abstract}
Copper/zinc superoxide dismutase (Cu/Zn SOD) is an essential enzyme that protects tissue from oxidative damage. Herein we report the first fluorogenic probe (SODO) for the detection and in vivo imaging of $\mathrm{Cu} / \mathrm{Zn}$ SOD. SODO represents a unique chemical probe for translational imaging studies of $\mathrm{Cu} / \mathrm{Zn}$ SOD in inflammatory disorders.
\end{abstract}

Superoxide dismutases (SOD, EC 1.15.1.1) are metalloenzymes that protect tissue from the oxidative stress caused by reactive oxygen species (ROS). ${ }^{1}$ The main function of SODs is to catalyse the dismutation of superoxide radicals $\left(\mathrm{O}_{2}{ }^{\bullet-}\right)$ to hydrogen peroxide $\left(\mathrm{H}_{2} \mathrm{O}_{2}\right)$ and oxygen. There are several isoforms of SODs, which can be distinguished by their metal cofactors and their distribution in cells. ${ }^{2}$ Among the different isoforms of SODs, copper/zinc superoxide dismutase (Cu/Zn SOD or SOD1) is widely distributed and comprises around $90 \%$ of the total SODs. Alterations in the expression and activity of $\mathrm{Cu} / \mathrm{Zn}$ SOD have been associated with the onset of a number of diseases. Mutations in human $\mathrm{Cu} / \mathrm{Zn}$ SOD are implicated in the development of neurological disorders, such as familial amyotrophic lateral sclerosis (fALS), Alzheimer's disease and Parkinson's disease. ${ }^{3-5}$ Furthermore, elevated activities of $\mathrm{Cu} / \mathrm{Zn}$ SOD have been reported in cancer (e.g. acute myelogenous leukaemia, Hodgkin's lymphoma) and chronic inflammatory diseases (e.g. rheumatoid arthritis, ischemic injury). ${ }^{5-7}$ On the

\footnotetext{
${ }^{a}$ Institute of Technical Biology and Agriculture Engineering, Key Laboratory of Ion Beam Bioengineering, Hefei Institutes of Physical Science, Chinese Academy of Sciences, Hefei, Anhui 230031, P. R. China. E-mail: zly0605@ustc.edu.cn

${ }^{b}$ Department of Chemistry, National University of Singapore, 3 Science Drive 2, 117543, Singapore

${ }^{c}$ Graduate School for Integrative Sciences and Engineering, National University of Singapore, Centre for Life Sciences, \#05-01, 28 Medical Drive, 117456 Singapore

${ }^{d}$ School of Life Sciences, University of Science and Technology of China, Hefei, Anhui 230027, P. R. China

${ }^{e}$ MRC Centre for Inflammation Research, Queen's Medical Research Institute, University of Edinburgh, EH16 4TJ Edinburgh, UK.

E-mail:mvendrel@staffmail.ed.ac.uk

$\dagger$ Electronic supplementary information (ESI) available: Structures and characterisation for all MK compounds. Full characterisation data (NMR, HR-MS) for all SODO derivatives. See DOI: 10.1039/c6cc00095a

\# Both authors contributed equally.
}

contrary, decreased levels of $\mathrm{Cu} / \mathrm{Zn}$ SOD have been associated with an inhibition of the immune response and the promotion of oxidative stress in age-related disorders. ${ }^{8,9}$

Despite the importance of $\mathrm{Cu} / \mathrm{Zn}$ SOD in regulating the balance between healthy and disease states, the exact mechanism that correlates $\mathrm{Cu} / \mathrm{Zn} \mathrm{SOD}$ to the progression of different pathologies remains largely unknown. ${ }^{5}$ Current probes to visualize SODs mainly rely on the intrinsic fluorescence of Tyr or Trp residues $^{10,11}$ or the use of non-specific metal chelators, such as bathocuproine. ${ }^{12}$ These methods have very limited practical use in vivo, due to spectral shortcomings (e.g. short excitation/emission wavelengths) and their poor selectivity between SODs and other ROS-related enzymes.

Fluorogenic probes are advantageous for in vivo imaging since they provide high signal-to-noise ratios without the need for washing steps. ${ }^{13,14}$ Our group and others have reported the preparation of fluorogenic probes based on the 4,4-difluoro-4bora-3a,4a-diaza-s-indacene (BODIPY) scaffold, ${ }^{15,16}$ one of the most exploited fluorophores for cell imaging due to its photostability and permeability properties. ${ }^{17,18}$ BODIPY fluorogens can be synthesized by direct conjugation of electron-rich groups (e.g. substituted benzene rings) to the BODIPY core, leading to photoinduced electron transfer (PeT) quenching and subsequent turn-on fluorescence emission in hydrophobic environments. In order to enhance the fluorogenic response of probes binding to $\mathrm{Cu}$ / Zn SOD, we designed a new class of BODIPY fluorogens combining PeT-quenching substituents and chemical groups restricting the rotational flexibility of the BODIPY core. The restriction of torsional motion has proven an effective strategy to generate turn-on fluorescent probes, ${ }^{19,20}$ and previous studies have shown that "-NH" groups directly linked to the position $\mathrm{C}_{3}$ of BODIPY can form intramolecular hydrogen bonds with the fluorine atoms. ${ }^{21}$ We synthesized MK fluorogens by modifying a 3,5-dichloroBODIPY scaffold (1) with benzylamines (M) forming intramolecular hydrogen bonds with the fluorine atoms, and triazole groups (K) as PeT-quenchers (Scheme 1). MK fluorogens were prepared by loading $\mathbf{1}$ onto 2-chlorotrityl chloride polystyrene (CTC-PS) resin, followed by nucleophilic substitution and 


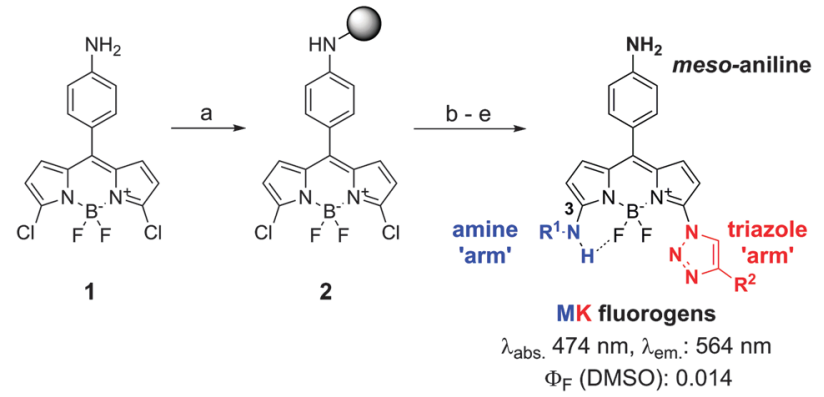

Scheme 1 Solid-phase synthesis of $\mathbf{M K}$ fluorogens. Reaction conditions: (a) CTC-PS, DIPEA, $\mathrm{CH}_{2} \mathrm{Cl}_{2}$ : DMF (1:1), r.t.; (b) $\mathrm{NaN}_{3}$, DMF, r.t.; (c) $\mathrm{R}^{1} \mathrm{NH}_{2}$, DIPEA : DMF (1:4), r.t.; (d) $\mathrm{R}^{2}-\mathrm{C} \equiv \mathrm{CH}$, Cul, L-ascorbic acid, r.t.; (e) TFA: $\mathrm{CH}_{2} \mathrm{Cl}_{2}(0.5: 99.5)$, r.t.

copper-catalysed azide-alkyne cycloaddition. A total of $40 \mathrm{MK}$ compounds with diverse amine and alkyne groups were isolated in moderate to high yields with very high purities using mild acidic cleavage conditions ${ }^{22}$ (for detailed chemical structures and characterisation data, see ESI $\dagger$ ).

The spectral characterisation of the MK derivatives confirmed their strong fluorogenic behaviour with minimal fluorescence in aqueous media, long Stokes shifts (i.e. around $90 \mathrm{~nm}$ ) and redshifted emission wavelengths when compared to the BODIPY core (Table S1 in ESI $\dagger$ ). As expected, the incorporation of benzylamines at the position $\mathrm{C}_{3}$ of the BODIPY scaffold restricted the torsional motion of the fluorophore, leading to an increase in the quantum yields in non-polar solvents. We also observed that the fluorescence emission of MK fluorogens correlated with solvent viscosity as a result of the decreased rotation of both triazole and aniline substituents (Fig. S1 in ESI $\dagger$ ). Moreover, the fluorogenic response of MK derivatives was stronger in non-polar solvents due to the reduced PeT quenching effect from the meso-aniline group in nonpolar environments (Fig. S2, S3 and Table S2 in ESI $\dagger$ ). Altogether, these results assert MK derivatives as BODIPY fluorogens with excellent spectral properties to detect polarity changes associated to the binding at large macromolecules.

In view of these properties, we assessed our MK fluorogens in vitro to bind at the macromolecular dimeric structure of $\mathrm{Cu} / \mathrm{Zn}$ SOD. The screening of diversity-oriented fluorescence libraries has become an effective strategy to identify highly selective molecular probes, ${ }^{23}$ and we observed that compounds with 2-ethoxybenzylamine (M103) as the amine group displayed high fluorescence emission after incubation with human $\mathrm{Cu} / \mathrm{Zn}$ SOD (hCu/Zn SOD) (Fig. S4 in ESI $\dagger$ ). MK103-48 showed the strongest response among all compounds and was selected for further studies (hereinafter named as SODO (SOD Orange), Fig. 1). SODO displayed up to 150 -fold increase in fluorescence emission after binding at $\mathrm{hCu} / \mathrm{Zn}$ SOD, with a limit of detection of $10 \mu \mathrm{g} \mathrm{mL}{ }^{-1}$ (Fig. S5 in ESI $\dagger$ ). Notably, SODO reached quantum yields around $45 \%$ and displayed a remarkable $60 \mathrm{~nm}$ hypsochromic shift in the fluorescence spectrum after binding (Fig. 1 and Fig. S5, S6 in ESI $\dagger$ ).

We examined the binding of SODO at $\mathrm{Cu} / \mathrm{Zn}$ SODs from different species. As with hCu/Zn-SOD, soDo displayed a concentration-dependent response in $\mathrm{Cu} / \mathrm{Zn}$ SODs from bovine

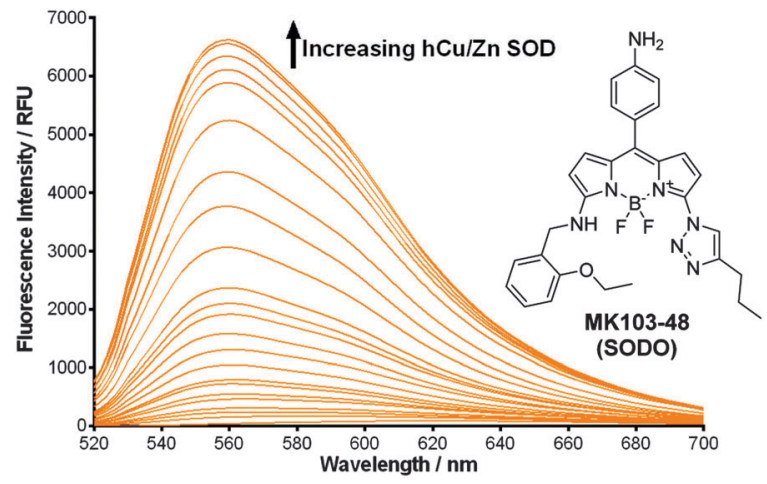

Fig. 1 Chemical structure and fluorescence spectra of SODO $(10 \mu \mathrm{M})$ after incubation with serial concentrations of hCu/Zn-SOD from 0.01 to $5 \mathrm{mg} \mathrm{mL}^{-1}$ in $20 \mathrm{mM}$ Tris- $\mathrm{HCl}$ buffer $(\mathrm{pH}=7.4)$. $\lambda_{\text {exc. }}: 460 \mathrm{~nm}$. $\Phi_{\mathrm{F}}$ in hCu/ Zn-SOD: 0.45 .

blood (bCu/Zn SOD) and from Arabidopsis thaliana $(\mathrm{aCu} / \mathrm{Zn}$ SOD) (Fig. 2). These results suggest that the binding of SODO is species-independent and occurs at a conserved hydrophobic region of $\mathrm{Cu} / \mathrm{Zn}$ SOD. While $\mathrm{Cu} / \mathrm{Zn}$ SOD stands for the majority of SOD in tissue, high selectivity for the $\mathrm{Cu} / \mathrm{Zn}$ SOD isoform is essential for imaging studies. We assessed the fluorescence response of SODO in the other two SOD isoforms (i.e. Mn-SOD and Fe-SOD) and observed high selectivity for $\mathrm{Cu} / \mathrm{Zn}$ SOD over Mn-SOD and Fe-SOD, where minimal binding was detected (Fig. 2). To the best of our knowledge, SODO is the first small fluorophore able to detect $\mathrm{Cu} / \mathrm{Zn}$ SOD with high specificity over other SODs. We also assessed the fluorescence emission of SODO in other ROS-related enzymes (e.g. catalase, peroxidase) (Fig. 2) and metabolites (e.g. $\mathrm{H}_{2} \mathrm{O}_{2}, \mathrm{O}_{2}{ }^{\bullet-},{ }^{1} \mathrm{O}_{2}, \mathrm{OH}^{\bullet}$ ) (Fig. 2, inset). SODO exhibited very high specificity for $\mathrm{Cu} / \mathrm{Zn}-\mathrm{SOD}$, showing minimal fluorescence in other enzymes and metabolites involved in oxidative damage and inflammatory processes. Finally we studied whether the binding of SODO affected the catalytic activity of $\mathrm{Cu} / \mathrm{Zn}$ SOD (Fig. S7 in ESI $\dagger$ ). SODO did not significantly perturb enzymatic function; hence being an

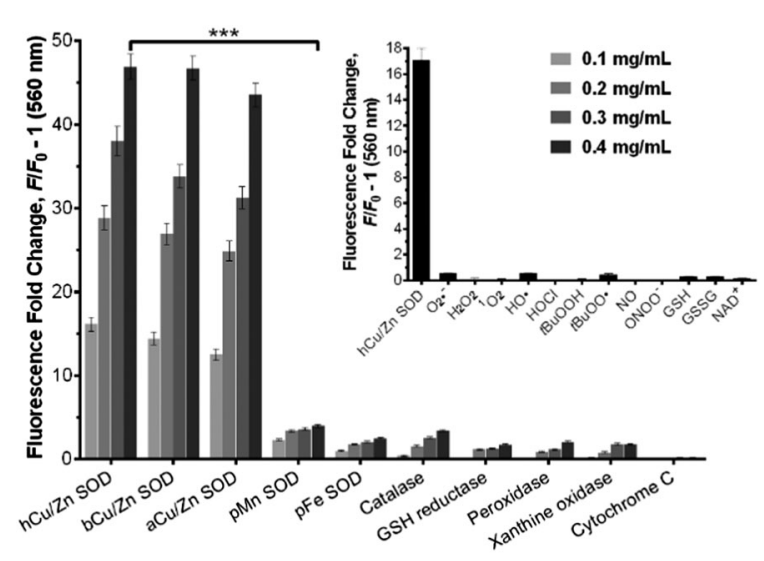

Fig. 2 Fluorogenic response of SODO upon binding to different proteins (at $0.1,0.2,0.3$ and $0.4 \mathrm{mg} \mathrm{mL}^{-1}$ ) and metabolites (inset, ROS \& RNS: $100 \mu \mathrm{M})$ in $20 \mathrm{mM}$ Tris- $\mathrm{HCl}$ buffer $(\mathrm{pH}=7.4)$. $\lambda_{\text {exc. }}: 460 \mathrm{~nm}, \lambda_{\mathrm{em}}: 560 \mathrm{~nm}$. Values are represented as means and error bars as standard deviations $(n=3),{ }^{* *}$ for $p<0.001$. 
excellent reporter of $\mathrm{Cu} / \mathrm{Zn} \mathrm{SOD}$ without altering the normal physiology of cells. Altogether, these results confirm SODO as the first fluorogenic probe to detect $\mathrm{Cu} / \mathrm{Zn}$ SOD without crossreacting with other SOD isoforms, enzymes or ROS.

In view of the high selectivity and fluorogenic properties of SODO, we employed it to visualise changes in the expression of $\mathrm{Cu} / \mathrm{Zn} \mathrm{SOD}$ in vivo. We used SODO to image $\mathrm{Cu} / \mathrm{Zn}$ SOD during the onset of inflammatory processes in zebrafish embryos. ${ }^{9} \mathrm{We}$ employed a zebrafish tail fin injury model of inflammation by amputating the tail fin of embryos at 3 days post fertilization (dpf), ${ }^{24}$ which allowed us to examine the in vivo fluorogenic response of SODO in the inflammatory milieu. As shown in Fig. 3b, zebrafish undergoing inflammation displayed bright fluorescence in the wound margins (white arrows), which correspond to inflamed areas where $\mathrm{Cu} / \mathrm{Zn}$ SOD is highly expressed. High magnification images corroborated the expression of $\mathrm{Cu} / \mathrm{Zn}$ SOD in the cytoplasm of epithelial cells (Fig. 3c). We further confirmed these results by measuring the levels of the sod1 gene before and after wounding using semi-quantitative reverse transcription-polymerase chain reaction (RT-PCR). As shown in Fig. 3d, the sod1 gene was highly upregulated $5 \mathrm{~h}$ after wounding, in agreement with the fluorescence emission profile of SODO in vivo (Fig. S8 in ESI $\dagger$ ). We also observed that SODO brightly stained oxidatively-stressed fibroblasts (Fig. S9 in ESI $\dagger$ ), containing high levels of $\mathrm{Cu} / \mathrm{Zn}$ SOD. ${ }^{25}$ Cell viability assays in fibroblasts also corroborated the marginal cytotoxicity of SODO within the working concentration range (Fig. S10 in ESI $\dagger$ ).
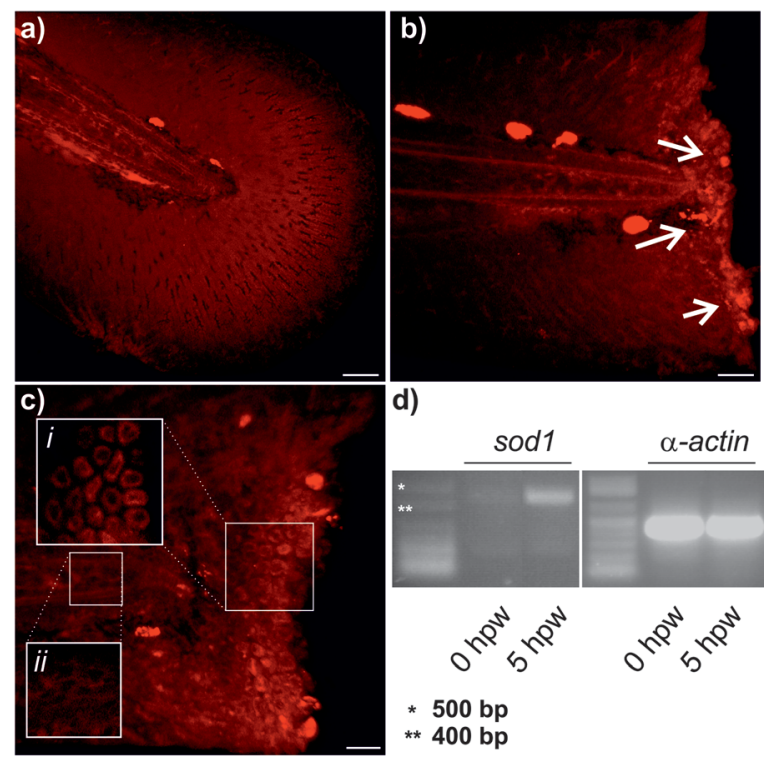

d)

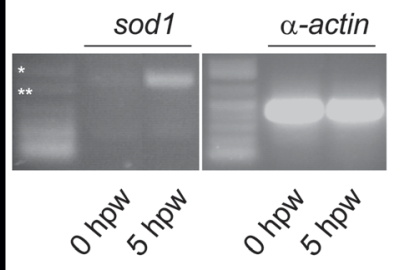

* $500 \mathrm{bp}$

** $400 \mathrm{bp}$

Fig. 3 In vivo imaging of $\mathrm{Cu} / \mathrm{Zn}$ SOD in inflamed zebrafish after treatment with SODO $(10 \mu \mathrm{M})$. (a) Unwounded tail fin of a zebrafish embryo (3 dpf). (b) tail fin of a zebrafish embryo ( $3 \mathrm{dpf}$ ) $5 \mathrm{~h}$ after wounding ( $5 \mathrm{hpw}$ ). Strong fluorescence emission is observed towards the wound margin (white arrows). Bright spots in (a) and (b) away from the wound edge correspond to auto-fluorescence signals from pigment cells. (c) High magnification images showing bright fluorescence from SODO at the wound margin (i) compared to non-fluorescent unwounded areas (ii). (d) Semi-quantitative RT-PCR of sod 1 and $\alpha$-actin genes at 0 and $5 \mathrm{hpw}$ with corresponding ladders. Scale bars (a and b): $40 \mu \mathrm{m}$; (c): $20 \mu \mathrm{m}$.
In order to determine the binding mode of SODO in $\mathrm{Cu} / \mathrm{Zn}$ SOD, we performed docking calculations to analyse the interaction between SODO at $\mathrm{hCu} / \mathrm{Zn}$ SOD. $\mathrm{Cu} / \mathrm{Zn}$-SOD is found in all eukaryotic species as a homodimeric enzyme of $\sim 32 \mathrm{kDa}$ containing one $\mathrm{Cu}$ and one $\mathrm{Zn}$ ion in each of the subunits, which are stabilized by an intra-chain disulfide bond. ${ }^{26}$ Our model predicted the interaction of SODO at the interface of the two subunits of $\mathrm{Cu} / \mathrm{Zn}$ SOD (Fig. 4a). The binding at this conserved hydrophobic pocket, which is away from the catalytic site of the enzyme, is consistent with the previously observed species-independent response of SODO (Fig. 2) and the fact that the enzymatic activity of $\mathrm{Cu} / \mathrm{Zn}$ SOD remained unaffected by SODO (Fig. S7 in ESI $\dagger$ ). A closer examination of the binding revealed four hydrogen bonds between SODO and hCu/Zn SOD: one hydrogen bond between the oxygen atom of the ethoxy group and Val148, two hydrogen bonds between the nitrogen atoms of the triazole ring and the residues Lys9 and Asn53, and a final hydrogen bond between the meso-aniline group and Asp11 (Fig. 4b). The binding analysis suggests that the fluorogenic response of SODO is the result of combining the restriction in the rotation of the fluorophore by forming four hydrogen bonds and the deactivation of the quenching PeT due to the migration to a hydrophobic environment, as observed in our results from the in vitro characterisation assays.

In order to corroborate this hypothesis, we prepared two derivatives of SODO lacking the chemical groups involved in the interaction with $\mathrm{hCu} / \mathrm{Zn}$ SOD (Fig. 5). We synthesized SODO1 as the derivative without the ethoxy group in the amine 'arm' and SODO2 as the derivative lacking the triazole nitrogen atoms (ESI $\dagger$ for synthetic details and characterisation), and compared their fluorogenic response to SODO after binding to hCu/Zn SOD. SODO1 and SODO2 showed remarkably lower fluorescence emission than SODO, confirming the relevance of both ethoxy and triazole groups for binding at hCu/Zn SOD (Fig. 5). These results confirmed the need of four hydrogen

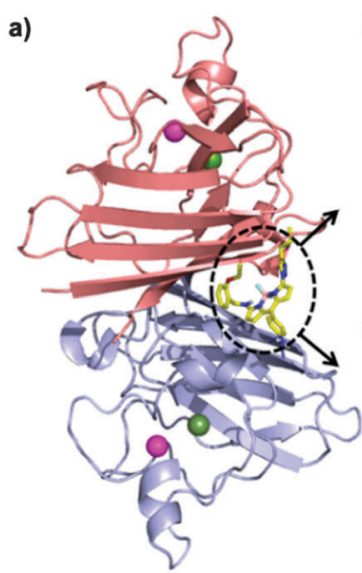

b)

Fig. 4 Molecular docking for the binding of SODO at hCu/Zn-SOD. (a) Illustration of the binding site of SODO (yellow) at the interface between the two monomeric subunits (blue and pink) of hCu/Zn-SOD (Cu and Zn are shown as green and magenta spheres, respectively). (b) Suggested hydrogen bonding interactions between SODO and different residues of hCu/Zn SOD. 

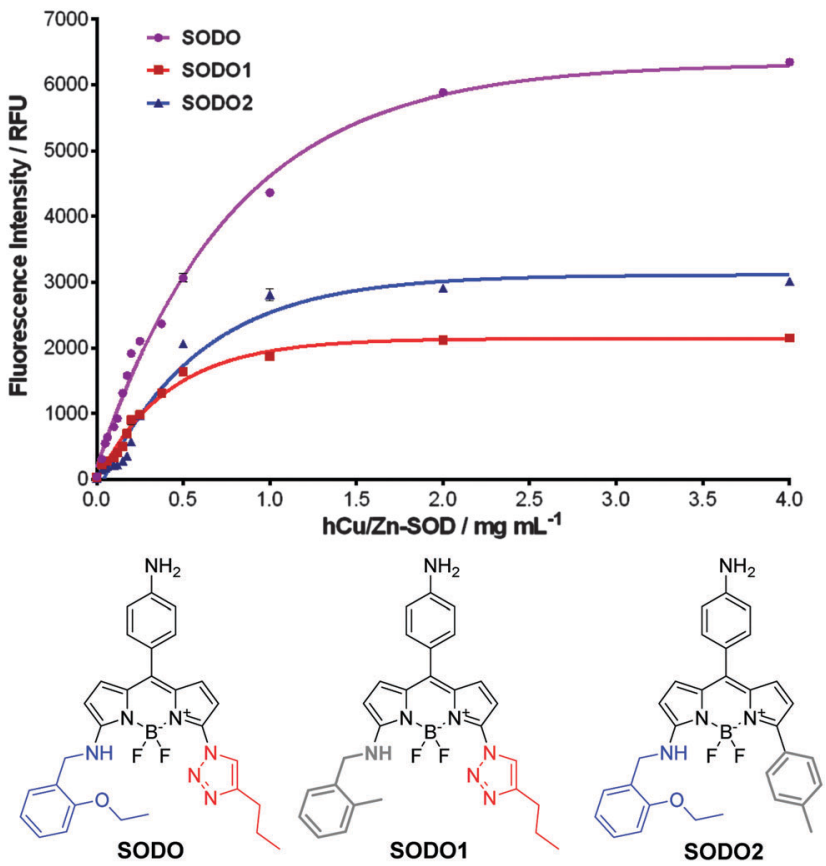

Fig. 5 Fluorogenic response of SODO derivatives upon incubation with serial concentrations of hCu/Zn SOD in $20 \mathrm{mM}$ Tris- $\mathrm{HCl}$ buffer $(\mathrm{pH}=7.4)$. $\lambda_{\text {exc: }}: 460 \mathrm{~nm}$ for SODO and SODO1, $510 \mathrm{~nm}$ for SODO2. $\Phi_{\mathrm{F}}$ in hCu/Zn SOD: SODO: 0.45, SODO1: 0.11, SODO2: 0.22 . Values are represented as means and error bars as standard deviations $(n=3)$.

bonds, which are missing in the analogues SODO1 and SODO2 (Fig. S11 in ESI $\dagger$ ), to restrict the torsional motion of SODO and induce its maximal fluorogenic response.

In summary, we have designed a new class of BODIPY fluorogens with enhanced spectral properties by incorporating both rotational restriction and PeT-quenching groups. These new BODIPY fluorogens show excellent properties as polarity probes with minimal background emission in aqueous media and long Stokes shifts upon fluorescence activation. In vitro studies identified one derivative (SODO) as a highly selective fluorogenic probe for $\mathrm{Cu} / \mathrm{Zn}$ SOD. SODO shows remarkable fluorescence emission only after binding to $\mathrm{Cu} / \mathrm{Zn}$ SOD with very high selectivity over ROS-related enzymes and metabolites as well as the other SOD isoforms (i.e. Mn-SOD and Fe-SOD). The high selectivity of SODO enabled its use for imaging $\mathrm{Cu} / \mathrm{Zn}$ SOD in vivo during the onset of an inflammatory response in a zebrafish tail fin injury model. Furthermore, we performed computational modelling to analyse the binding of SODO at $\mathrm{Cu} / \mathrm{Zn}$ SOD. Structure-activity studies suggest that the binding occurs at the interface of the two enzymatic subunits and involves four residues to restrict the torsional motion of the BODIPY fluorophore and deactivate its PeT-quenching groups. SODO is the first fluorogenic probe for $\mathrm{Cu} / \mathrm{Zn}$ SOD and represents a unique probe for the detection and in vivo imaging of $\mathrm{Cu} / \mathrm{Zn} \mathrm{SOD}$ during the progression of inflammatory disorders.

L. Z. acknowledges the '973' program (2014CB932002), the Natural Science Foundation of China (11105150) and the Special Financial Grant from China Postdoctoral Science Foundation (2013T60613). J. C. E. acknowledges a NGS scholarship. Y. F. is a Wellcome Trust Sir Henry Dale Fellow (WT 100104/Z/12/Z). Y.-T. C. acknowledges funding from the National Medical Research Council (NMRC/CBRG/0015/2012). M. V. acknowledges funding from Medical Research Council, Marie Curie Career Integration Grant (333487) and the WT Institutional Strategic Support Fund.

\section{References}

1 I. Fridovich, Annu. Rev. Biochem., 1995, 64, 97.

2 I. N. Zelko, T. J. Mariani and R. J. Folz, Free Radical Biol. Med., 2002, 33, 337.

3 H. J. Lee, K. J. Korshavn, A. Kochi, J. S. Derrick and M. H. Lim, Chem. Soc. Rev., 2014, 43, 6672.

4 M. A. Hough, J. G. Grossmann, S. V. Antonyuk, R. W. Strange, P. A. Doucette, J. A. Rodriguez, L. J. Whitson, P. J. Hart, L. J. Hayward, J. S. Valentine and S. S. Hasnain, Proc. Natl. Acad. Sci. U. S. A., 2004, 101, 5976.

5 R. Noor, S. Mittal and J. Iqbal, Med. Sci. Monit., 2002, 8, 210.

6 R. Gonzales, C. Auclair, E. Voisin, H. Gautero, D. Dhermy and P. Boivin, Cancer Res., 1984, 44, 4137.

7 L. Papa, G. Manfredi and D. Germain, Genes Cancer, 2014, 5, 15.

8 A. Vasilaki and M. J. Jackson, Free Radical Biol. Med., 2013, 65, 317.

9 M. Marikovsky, V. Ziv, N. Nevo, C. Harris-Cerruti and O. Mahler, J. Immunol., 2003, 170, 2993.

10 S. T. Ferreira, L. Stella and E. Gratton, Biophys. J., 1994, 66, 1185.

11 E. Luchinat, A. Gianoncelli, T. Mello, A. Galli and L. Banci, Chem. Commun., 2015, 51, 584.

12 D. V. Martyshkin, S. B. Mirov, Y. X. Zhuang, J. P. Crow, V. Ermilov and J. S. Beckman, Spectrochim. Acta, Part A, 2003, 59, 3165.

13 P. Shieh, V. T. Dien, B. J. Beahm, J. M. Castellano, T. Wyss-Coray and C. R. Bertozzi, J. Am. Chem. Soc., 2015, 137, 7145.

14 Y. Hori, T. Norinobu, M. Sato, K. Arita, M. Shirakawa and K. Kikuchi, J. Am. Chem. Soc., 2013, 135, 12360.

15 J. C. Er, M. K. Tang, C. G. Chia, H. Liew, M. Vendrell and Y.-T. Chang, Chem. Sci., 2013, 4, 2168.

16 H. Sunahara, Y. Urano, H. Kojima and T. Nagano, J. Am. Chem. Soc., 2007, 129, 5597.

17 T. Kowada, H. Maeda and K. Kikuchi, Chem. Soc. Rev., 2015, 44, 4953-4972.

18 A. Vázquez-Romero, N. Kielland, M. J. Arévalo, S. Preciado, R. J. Mellanby, Y. Feng, R. Lavilla and M. Vendrell, J. Am. Chem. Soc., 2013, 135, 16018.

19 Y.-H. Ahn, J.-S. Lee and Y.-T. Chang, J. Comb. Chem., 2008, 10, 376.

20 Y. Urano, M. Kamiya, K. Kanda, T. Ueno, K. Hirose and T. Nagano, J. Am. Chem. Soc., 2005, 127, 4888.

21 W. Qin, V. Leen, T. Rohand, W. Dehaen, P. Dedecker, M. van der Auweraer, K. Robeyns, L. van Meervelt, D. Beljonne, B. van Averbeke, J. N. Clifford, K. Driesen, K. Binnemans and N. l. Boens, J. Phys. Chem. A, 2008, 113, 439.

22 M. Vendrell, G. G. Krishna, K. K. Ghosh, D. Zhai, J.-S. Lee, Q. Zhu, Y. H. Yau, S. G. Shochat, H. Kim, J. Chung and Y.-T. Chang, Chem. Commun., 2011, 47, 8424.

23 J.-S. Lee, M. Vendrell and Y.-T. Chang, Curr. Opin. Chem. Biol., 2011, $15,760$.

24 L. Li, B. Yan, Y. Q. Shi, W. Q. Zhang and Z. L. Wen, J. Biol. Chem., 2012, 287, 25353.

25 J. H. Chen, K. Stoeber, S. Kingsbury, S. E. Ozanne, G. H. Williams and C. N. Hales, J. Biol. Chem., 2004, 279, 49439.

26 J. A. Tainer, E. D. Getzoff, K. M. Beem, J. S. Richardson and D. C. Richardson, J. Mol. Biol., 1982, 160, 181. 